\title{
On the modeling of MMC for use in large scale dynamic simulations
}

\author{
Julian Freytes*, Lampros Papangelis ${ }^{\dagger}$, Hani Saad ${ }^{\ddagger}$, Pierre Rault ${ }^{\ddagger}$, Thierry Van Cutsem $^{\S}$ and Xavier Guillaud ${ }^{*}$ \\ ${ }^{*}$ Ecole Centrale de Lille, L2EP †University of Liège \\ Lille, France \\ Belgium \\ $\begin{array}{cl}{ }^{\ddagger} \text { RTE } & { }^{\S} \text { Fund for Scientific Research } \\ \text { La Défense, France } & \text { University of Liège, Belgium }\end{array}$
}

\begin{abstract}
This paper focuses on simplified models of the Modular Multilevel Converter suitable for large-scale dynamic studies, in particular simulations under the phasor approximation. Compared to the existing literature, this paper does not a priori adopt the modeling approach followed for the original twolevel or three-level Voltage Source Converter. On the contrary, a model is derived following a physical analysis that preserves its average internal dynamic behavior. An equivalent control structure is proposed and various alternatives are highlighted. The proposed model with its controllers has been implemented in a phasor simulation software and its response has been validated against a detailed Electromagnetic Transient model. Finally, an illustrative example is presented with the application of the proposed model on a large grid consisting of $\mathrm{AC}$ areas interconnected with a multi-terminal DC grid.
\end{abstract}

Index Terms-High Voltage Direct Current (HVDC), Modular Multilevel Converter (MMC), Voltage Source Converter (VSC), Phasor approximation.

\section{INTRODUCTION}

Modular Multilevel Converters (MMC) are currently the state of the art in High Voltage Direct Current (HVDC) transmission. Their superior performance in terms of power rating, losses and harmonics generation, compared to the original twoand three- level Voltage Source Converters (VSC), is expected to make the MMC topology the default option for future HVDC systems [1]. Moreover, MMC is currently considered as the best option for the envisioned implementation of MultiTerminal DC (MTDC) grids.

This motivates the development of a simplified MMC model, which can be used for simulations under the phasor approximation in order to facilitate large-scale studies.

A phasor MMC model was first described in [2]. A number of recent publications have adopted the same modeling approach for the MMC as for the two or three-level VSC, though with small modifications. However, these models are either based on strong assumptions regarding the way the MMC control is implemented [3], [4] or they introduce supplementary functions and resort to parameter identification techniques to reproduce its dynamic behavior [5]. Moreover, this type of modeling may not be fully satisfactory since it tends to neglect the significant structural differences between both topologies.

Another simplified MMC model is presented in [6], which has been developed including the circulating current suppress- ing controller. However, the inputs of the MMC model are imposed and they are not a result of the control system.

In this paper, an MMC simplified model is derived following a physical analysis of the converter. The aim is to maintain the average internal dynamics of the MMC, which do not exist in the two-level VSC, i.e. the dynamics of the DC current and of the energy stored. For this reason, some assumptions are made regarding the way the energy is stored inside the MMC and an equivalent electrical circuit is derived. In addition, a control structure inherited from the simplified model, equivalent to the control structure of the EMT model, is described. A similar approach has been described in [7], but the paper focuses on the small-signal modeling of an MMC, and not on its integration in large scale dynamic simulations.

The resulting simplified model along with its controllers has been implemented in a phasor simulation software and its response has been validated against a detailed reference EMT model. Simulation results validate the effectiveness of the proposed modeling approach in capturing the average behavior of the MMC. Furthermore, the model has been used in the simulations of an MTDC grid interconnecting two asynchronous AC areas.

The rest of the paper is organized as follows. Section II presents the derivation of the MMC simplified model. Its validation using a point-to-point HVDC link is reported in Section III. Section IV presents results of application in an MTDC grid. Finally, conclusions are provided in Section V.

\section{MMC Simplified MODEL DERIVATION}

\section{A. Arm Average Model (AAM)}

The starting point of the derivation is the AAM, a simplification of the detailed MMC model that has been proposed in order to accelerate the simulation of MMC EMT models and investigate different control strategies [8].

The topology of the AAM is recalled in Fig. 1. There is one leg for each phase $a, b, c$, each consisting of an upper and a lower arm. This model assumes that the voltages of all the submodule capacitors are maintained in a close range, thus allowing to replace them by an equivalent one. Therefore, each arm includes an arm inductance $L_{\text {arm }}$, an arm resistance $R_{\text {arm }}$ and an equivalent capacitor $C_{t o t}$ in parallel with a chopper. This model is suitable when the focus is on the AC and DC current dynamics and the total energy stored in each arm. 


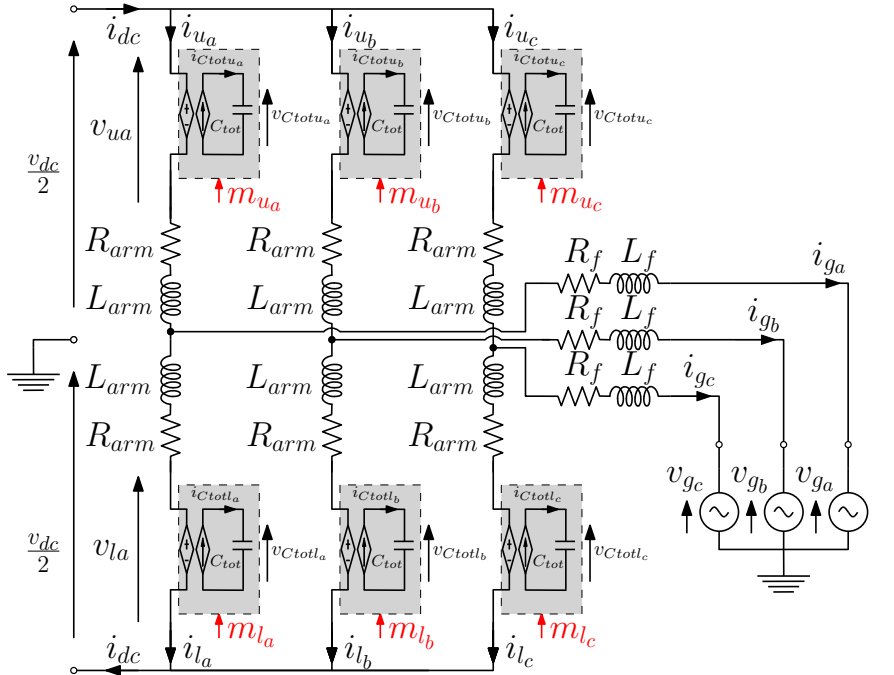

Figure 1: MMC Arm average model

The voltages $v_{m u_{j}}\left(v_{m l_{j}}\right)$ and currents $i_{u_{j}}\left(i_{l_{j}}\right)$ of each arm $j(j=a, b, c)$ are described by the following equations:

$$
\begin{gathered}
v_{m u_{j}}=m_{u_{j}} v_{C t o t u_{j}}, \quad v_{m l_{j}}=m_{l_{j}} v_{C t o t l_{j}} \\
i_{C t o t u_{j}}=m_{u_{j}} i_{u_{j}}, \quad i_{C t o t l_{j}}=m_{l_{j}} i_{l_{j}}
\end{gathered}
$$

where $v_{C t o t u_{j}}\left(v_{C t o t l_{j}}\right)$ is the voltage across the upper (lower) arm equivalent capacitor, $m_{u_{j}}\left(m_{l_{j}}\right)$ is the corresponding instantaneous duty cycle and $i_{C t o t u_{j}}\left(i_{C t o t l_{j}}\right)$ is the current through the upper (lower) arm capacitor. The voltage and current of the equivalent capacitor are related through the capacitor equation:

$$
i_{C t o t u_{j}}=C_{t o t} \frac{d v_{C t o t u_{j}}}{d t}, \quad i_{C t o t l_{j}}=C_{t o t} \frac{d v_{C t o t l_{j}}}{d t} .
$$

At this stage, the MMC is characterized by 11 independent state variables: the six equivalent capacitor voltages and five currents (three arm currents and two phase currents).

Applying Kirchhoff's law, the following equations are derived for each phase $j$ :

$\frac{v_{d c}}{2}-v_{m u_{j}}-L_{a r m} \frac{d i_{u_{j}}}{d t}-R_{a r m} i_{u_{j}}-L_{f} \frac{d i_{g_{j}}}{d t}-R_{f} i_{g_{j}}-v_{g_{j}}=0$ (4)

$-\frac{v_{d c}}{2}+v_{m l_{j}}+L_{a r m} \frac{d i_{l_{j}}}{d t}+R_{a r m} i_{l_{j}}-L_{f} \frac{d i_{g_{j}}}{d t}-R_{f} i_{g_{j}}-v_{g_{j}}=0$. (5)

The addition of (4) and (5) yields:

$$
v_{v_{j}}-v_{g_{j}}=L_{e q}^{a c} \frac{d i_{g_{j}}}{d t}+R_{e q}^{a c} i_{g_{j}}
$$

where:

$$
\begin{gathered}
i_{g_{j}}=i_{u_{j}}-i_{l_{j}} \\
v_{v_{j}}=\frac{-v_{m u_{j}}+v_{m l_{j}}}{2} \\
R_{e q}^{a c}=\left(R_{a r m}+2 R_{f}\right) / 2, \quad L_{e q}^{a c}=\left(L_{a r m}+2 L_{f}\right) / 2 .
\end{gathered}
$$

Equation (6) describes the AC side dynamics of the AAM.
The subtraction of (4) and (5) gives:

$$
v_{d c}-v_{d i f f_{j}}=2 L_{a r m} \frac{d i_{d i f f_{j}}}{d t}+2 R_{a r m} i_{d i f f_{j}}
$$

where the differential current $i_{\text {diff }}$ and voltage $v_{\text {diff }}$ are defined as:

$$
\begin{gathered}
i_{d i f f_{j}}=\frac{i_{u_{j}}+i_{l_{j}}}{2} \\
v_{d i f f_{j}}=v_{m u_{j}}+v_{m l_{j}} .
\end{gathered}
$$

Equation (10) describes the DC side dynamics of the AAM.

\section{B. Simplification and AAM equivalent circuit}

In normal operation, the control system keeps $v_{C t o t u_{j}}^{2}$ close to $v_{\mathrm{Ctotl}_{j}}^{2}$ [3], allowing to consider:

$$
v_{C t o t u_{j}}=v_{C t o t l_{j}}=\bar{v}_{C t o t_{j}}
$$

where $\bar{v}_{C t o t_{j}}$ is the average of the upper and lower arm capacitor voltages of each arm of the MMC, i.e. $\bar{v}_{C t o t_{j}}=$ $\left(v_{C_{\text {totu }}}+v_{\text {Ctotl }}\right) / 2$.

The combinations of (13) with (2) and (3), and the summation of upper and lower arm equations yields:

$$
2 C_{t o t} \frac{d \bar{v}_{C t o t_{j}}}{d t}=m_{u_{j}} i_{u_{j}}+m_{l_{j}} i_{l_{j}}
$$

At this point, two new AC and DC duty cycles are defined as follows:

$$
\begin{aligned}
m_{a c_{j}} & =\frac{\left(m_{l_{j}}-m_{u_{j}}\right)}{2} \\
m_{d c_{j}} & =\left(m_{u_{j}}+m_{l_{j}}\right) .
\end{aligned}
$$

Taking into account (13), and substituting (15) and (16) in (8) and (12) yields:

$$
\begin{gathered}
v_{v_{j}}=m_{a c_{j}} \bar{v}_{C t o t_{j}} \\
v_{d i f f_{j}}=m_{d c_{j}} \bar{v}_{C_{t o t}} .
\end{gathered}
$$

Equation (14) is further combined with (11), (15), and (16), resulting in:

$$
2 C_{t o t} \frac{d \bar{v}_{C t o t_{j}}}{d t}=m_{d c_{j}} i_{d i f f_{j}}-m_{a c_{j}} i_{g_{j}}=i_{m d c, j}-i_{m a c, j}
$$

where the modulated DC and AC currents are introduced:

$$
i_{m d c, j}=m_{d c_{j}} i_{d i f f_{j}}, \quad i_{m a c, j}=m_{a c_{j}} i_{g_{j}} .
$$

Equations (6), (10), and (19) can be combined into the equivalent circuit of Fig. 2 for each phase [9].

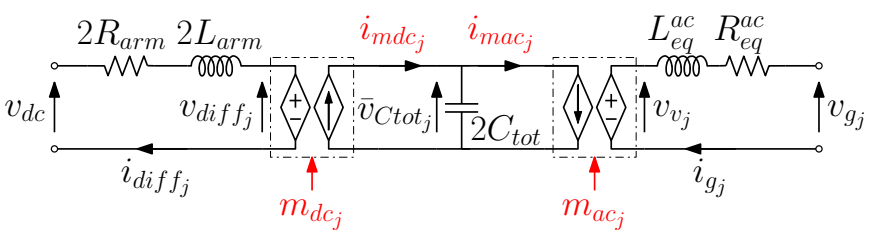

Figure 2: Equivalent circuit of each AAM phase 


\section{Simplification under balanced conditions}

In normal operation, the MMC control system distributes the total energy stored in the converter equally between each leg. Therefore, in order to further simplify the model for a balanced AC system, it is reasonable to assume:

$$
\bar{v}_{C t o t_{a}}=\bar{v}_{C t o t_{b}}=\bar{v}_{C t o t_{c}}=\bar{v}_{C t o t} .
$$

Following this assumption, summing the Eqs. (19) over the three phases results in:

$$
C_{e q} \frac{d \bar{v}_{C t o t}}{d t}=\sum_{j=a, b, c} i_{m d c_{j}}-\sum_{j=a, b, c} i_{m a c_{j}} .
$$

As can be seen, the assumption in (21) allows replacing all six arm capacitors by an equivalent capacitance $C_{e q}=6 C_{t o t}$ subject to voltage $\bar{v}_{C t o t}$. Figure 2 and Eq. (22) also show that the charging or discharging of that equivalent capacitor depends on the difference between the sum of the modulated DC currents and the sum of the modulated AC currents.

An additional assumption can be made in order to further simplify (22). If it is also assumed that $i_{d c}$ is distributed equally among the three legs of the MMC (i.e. $m_{d c}=m_{d c_{a}}=$ $m_{d c_{b}}=m_{d c_{c}}$ ), and taking into account that $\sum i_{d i f f_{j}}=i_{d c}$, the first sum in the right-hand side of (22) is equal to:

$$
\sum_{j=a, b, c} i_{m d c_{j}}=i_{d c} m_{d c} .
$$

As far as the second sum in (22) is concerned, using (17) and (21), it can be expressed as:

$$
\sum_{j=a, b, c} i_{m a c_{j}}=\frac{1}{\bar{v}_{C t o t}} \sum_{j=a, b, c} v_{v_{j}} i_{g_{j}}=\frac{p_{m a c}}{\bar{v}_{C t o t}}
$$

where $p_{\operatorname{mac}}$ is the instantaneous three-phase $\mathrm{AC}$ power flowing out of the MMC.

In phasor mode simulation, balanced conditions are usually assumed. Therefore, after transformation to the $d q$ reference frame, $p_{m a c}$ can be rewritten as:

$$
p_{m a c}=v_{m d} i_{i g d}+v_{m q} i_{i g q}=\left(m_{d} i_{i g d}+m_{q} i_{i g q}\right) \bar{v}_{C t o t}
$$

where the subscripts $d$ and $q$ indicate the $d$-axis and $q$-axis components of the corresponding variables and $m_{d}, m_{q}$ are defined as:

$$
m_{d}=\frac{v_{m d}}{\bar{v}_{C t o t}}, \quad m_{q}=\frac{v_{m q}}{\bar{v}_{C t o t}} .
$$

As a result, (22) becomes:

$$
C_{e q} \frac{d \bar{v}_{C t o t}}{d t}=m_{d c} i_{d c}-m_{d} i_{g d}-m_{q} i_{g q} .
$$

Furthermore, the AC dynamics equation (6) can be decomposed into:

$$
\begin{aligned}
& v_{m d}-v_{g d}=L_{e q}^{a c} \frac{d i_{g d}}{d t}+R_{e q}^{a c} i_{g d}-\omega L_{e q}^{a c} i_{g q} \\
& v_{m q}-v_{g q}=L_{e q}^{a c} \frac{d i_{g q}}{d t}+R_{e q}^{a c} i_{g q}+\omega L_{e q}^{a c} i_{g d} .
\end{aligned}
$$

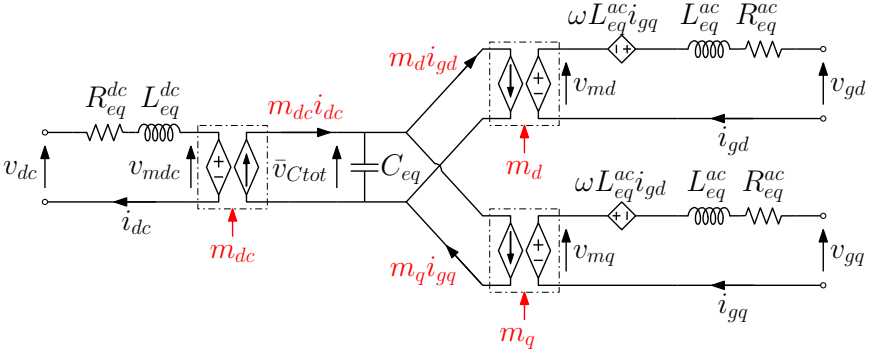

(a) MMC circuit model in $d q$-frame

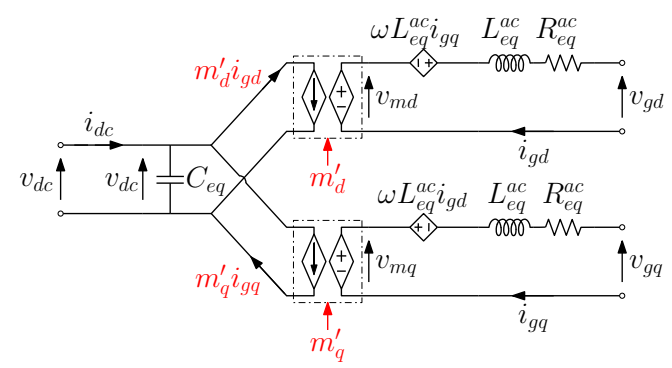

(b) Classical VSC model in $d q$-frame

Figure 3: Equivalent circuits of MMC and two-level VSC

Finally, the DC-side equation of the MMC (10) is simplified as follows:

$$
v_{d c}-v_{m d c}=L_{e q}^{d c} \frac{d i_{d c}}{d t}+R_{e q}^{d c} i_{d c}
$$

where:

$$
\begin{gathered}
v_{m d c}=m_{d c} \bar{v}_{C t o t} \\
R_{e q}^{d c}=\frac{2 R_{a r m}}{3}, \quad L_{e q}^{d c}=\frac{2 L_{a r m}}{3} .
\end{gathered}
$$

Following the above analysis, a new equivalent circuit for the MMC model based on (27), (28), (29) and (30) is derived as shown in Fig. 3a.

When comparing the equivalent model shown in Fig. 3a to the AAM of Fig. 1, it can be seen that the total number of independent variables has been reduced from 11 to 4 . This reduction has been achieved due to the replacement of the six equivalent capacitor voltages $\left(v_{\mathrm{Ctotu}_{j}}, v_{\mathrm{Ctotl} l_{j}}\right.$ in Fig. 1) by a single equivalent $\bar{v}_{C t o t}$. Also, the three differential currents $i_{d i f f_{j}}$ have been combined in only one DC current $i_{d c}$.

\section{Comparison with a two-level VSC phasor model}

In Fig. 3 the proposed equivalent circuit (Fig. 3a) is juxtaposed with the equivalent circuit of a classical two-level VSC model in $d q$ frame (Fig. 3b) similar to the one proposed in [4] and [5] in order to represent an MMC. It can be seen that in both representations, the AC-side is identical.

However, since the capacitor in the representation as a twolevel VSC is connected directly to the DC side, the connection between $\mathrm{AC}$ and $\mathrm{DC}$ sides can be found using the following equation:

$$
C_{e q} \frac{d v_{d c}}{d t}=i_{d c}-m_{d}^{\prime} i_{g d}-m_{q}^{\prime} i_{g q}
$$


where $m_{d}^{\prime}$ and $m_{q}^{\prime}$ are in this case defined as:

$$
m_{d}^{\prime}=\frac{v_{m d}}{v_{d c}}, \quad m_{q}^{\prime}=\frac{v_{m q}}{v_{d c}} .
$$

It can be observed that (33) is similar to (27) with the substitution of $\bar{v}_{C t o t}$ by $v_{d c}$.

A direct consequence of the above is that the DC current is not an independent state variable in the two-level VSC model as it is in the MMC model. Therefore, it cannot be controlled in the former as it can in the latter.

An even more significant difference is that the DC capacitor of a classical VSC is physically coupled with the HVDC system, while, in contrast, the total capacitance of the MMC can be decoupled from it [3]. This in turn, can have significant impact on the DC voltage response since the total capacitance connected to an HVDC system may be greatly reduced [10].

Moreover, $R_{e q}^{d c}$ is not present in the model of the two-level VSC. Therefore, in order to have the same losses in the two models of Fig. 3, $R_{e q}^{d c}$ should be added to its DC side.

Note finally that the classical VSC model in Fig. $3 \mathrm{~b}$ can be directly derived from the proposed MMC model of Fig. 3a by setting the derivative terms in (27) and (30) equal to zero, and connecting the capacitance $C_{e q}$ directly to the DC bus.

\section{E. MMC Control}

In this section a control structure, equivalent to the control of the AAM described in [11], is presented for the simplified MMC model. As in the two-level VSC, a control strategy based on two cascaded loops (namely inner and outer loops) is proposed. The inner loops control the AC and DC currents, while the outer loops generate the current references [12].

It has to be noted that the $d q$ reference frame to which all $\mathrm{AC}$ currents and voltages are referred has to be provided by a Phase-Lock-Loop (PLL), which aims at aligning the grid voltage phasor $v_{g}$ with the $d$ axis.

The inner control loops are shown in Fig. 4. Two PI controllers are used to control the currents $i_{g d}$ and $i_{g q}$ to their references $i_{g d, r e f}$ and $i_{g q, r e f}$, respectively, and decoupling terms are introduced to achieve independent active and reactive power control. Similarly, a PI controller is used for the control of the DC current. In all cases, the modulated voltage outputs $v_{m d}, v_{m q}$ and $v_{m d c}$ are divided by the equivalent capacitor voltage $\bar{v}_{c t o t}$ to produce the control outputs $m_{d}, m_{q}$ and $m_{d c}$, respectively. The response time of these controllers is usually in the order of a few milliseconds. In the following, the AC and DC current loops have been tuned to achieve a response time of $5 \mathrm{~ms}$ and $4 \mathrm{~ms}$, respectively.

The current references in Fig. 4 are generated by the outer controllers shown in Fig. 5. Several control strategies can be implemented for the components $i_{g d, r e f}$ and $i_{g q, r e f}$. Figure 5 illustrates a subset of those strategies, namely a choice between active power and DC voltage control for $i_{g d, r e f}$, and constant reactive power for $i_{g q, r e f}$. The power control consists of an open loop controller that provides $i_{g d, r e f}$ as the ratio between the reference AC active power $P_{a c, r e f}$ and the terminal AC voltage magnitude $v_{g d}$. Another option could be a combination

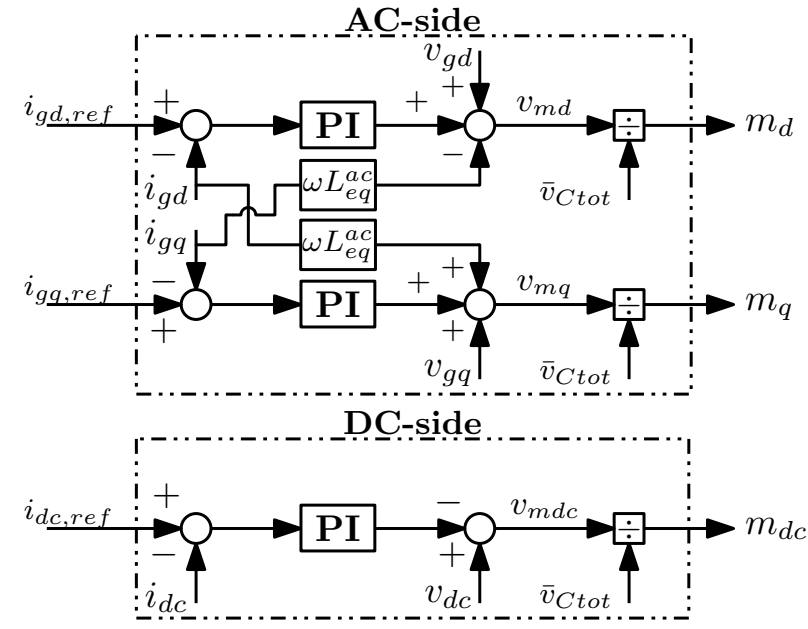

Figure 4: Inner control loops

of integral and open loop control. Moreover, $P_{a c, r e f}$ can be either constant or it can follow a $P_{a c}-v_{d c}$ droop characteristic. The DC voltage control is implemented with a PI controller. As far as reactive power $\left(Q_{a c}\right)$ control is concerned, the case with an integral control is shown.

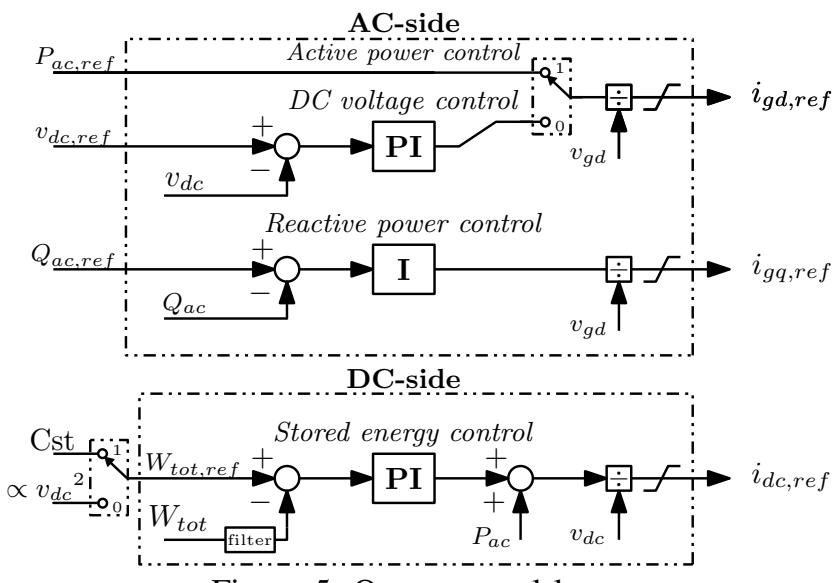

Figure 5: Outer control loops

The DC current reference is the output of the total energy control loop. The control of the total energy $\left(W_{t o t}\right)$ is based on the regulation of the exchanged energy between $\mathrm{AC}$ and DC sides [3]. A PI controller is used to bring the total stored energy near the reference $W_{\text {tot,ref }}$. The latter can be a constant value as considered in [3] (upper position of the switch in Fig. 5) or it can be associated with the square of the DC voltage $v_{d c}^{2}$ as discussed in [11] (lower position of the same switch). The latter allows sharing the MMC stored energy with the DC bus. Finally, it is common to add an AC power compensation $P_{a c}$ as well as a filter on the MMC energy signal before introducing it into the control [11].

The outer controllers are usually tuned to be much slower than the inner current loops to avoid undesired interactions. A common practice is to tune the outer loops at least ten times slower. In the following, the power, DC voltage and energy controllers have been tuned to achieve a response time of 100 $\mathrm{ms}, 70 \mathrm{~ms}$ and $50 \mathrm{~ms}$, respectively. 
At this point, it is highlighted that if a classical two-level VSC model is chosen to represent the MMC, the state variables of the DC current and the total energy are eliminated and, thus, the corresponding control loops cannot be taken into account.

\section{Simplified MMC MOdEL VALIDATION}

Figure 6 shows the HVDC point-to-point test case system used to validate the proposed simplified model. MMC-1 is chosen to control the power flowing in the HVDC link, while MMC-2 regulates the DC voltage at its terminal. The $70-\mathrm{km}$ DC cable is represented with a $\Pi$-model consisting only of resistive and capacitive elements. The parameters of the MMCs and the cable are listed in the Appendix. Both AC sides are modeled as Thévenin equivalents with a Short Circuit Ratio (SCR) equal to 10 . The simplified model along with its controllers has been implemented in RAMSES, a research simulation software developed at the University of Liège [13], [14]. The reference for the validation is the so-called Model \#2 ,i.e. equivalent circuit based model in [8], or Model \#4 in [15], of a 401-level converter implemented in the EMTPRV software [16]. The control structure used in the reference model is equivalent to the one presented for the simplified model.

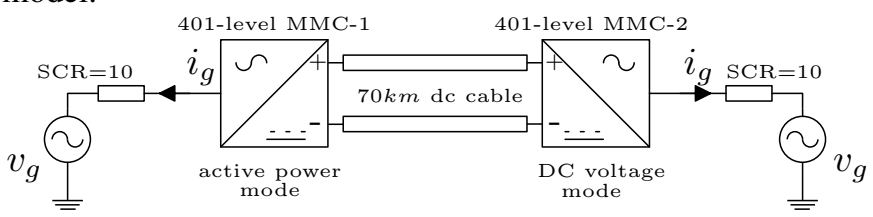

Figure 6: HVDC point-to-point system

Starting with a power transfer of $0.3 \mathrm{pu}$ (MMC-1), the simulated events are listed as follows:

- at $t=0.4 \mathrm{~s}$, a step of $-0.4 \mathrm{pu}$ is applied to the AC power reference of MMC-1,

- at $t=0.8 \mathrm{~s}$, a step of $0.01 \mathrm{pu}$ is applied to the DC voltage reference of MMC-2.

The reactive power reference $Q_{a c, r e f}$ of both MMCs is equal to zero throughout the simulation. Results of the validation are shown only with the MMCs controlled to share their stored energy with the energy of the cable capacitance, i.e. the lower switch in Fig. 5 is in the lower position.

The active power of MMC-1 is shown in Fig. 7. The average behavior of the reference model is very satisfactorily reproduced. The small difference during the transient is negligible.

The power reference step temporarily creates an imbalance in the HVDC link resulting in a change of the DC voltage. Fig. 8 shows the DC voltage response at MMC-2 terminal. It can be seen that the response of the simplified model matches very accurately the reference response. The evolution of the total energy $W_{\text {tot }}$ stored in MMC-1 is shown in Fig. 9. The response is similar to the DC voltage response, which can be attributed to the MMCs control strategy of sharing their energy with the DC link.

Lastly, the assumptions made in order to derive the simplified model, namely $i_{\text {diff } f_{a}}=i_{\text {diff } f_{b}}=i_{\text {diff } f_{c}}=i_{d c} / 3$ and $v_{C_{\text {Ctotu }}}=v_{C t o t l_{j}}=\bar{v}_{C t o t}$, are tested in Figs. 10, 11 and 12 (for the phase $a$ only). For all cases, the simplified model reproduces very accurately the average response of the reference model.

\section{MTDC GRID CASE STUDY}

\section{A. MTDC grid test system}

In this section, results are presented of the application of the simplified model in a large system. Moreover, a comparison is conducted between three different cases regarding the MMC model and control.

A simplified representation of the combined AC and DC system is shown in Fig. 13. It consists of two AC areas and an offshore wind farm interconnected through an MTDC grid. Each AC system is a variant of the Nordic test system detailed in [17] and includes a total of 76 buses and 22 synchronous machines modeled in detail with their regulators. The two AC systems are identified as "Nordic West" and "Nordic East". The wind farm is modeled as a constant power injection. There are five MMC terminals in total, of which two are connected to each $\mathrm{AC}$ system and one to the wind farm.

Regarding the HVDC cables, the same representation as in Section III is used. Their parameters are given in Table I. The initial operating point of the MTDC grid is given in Table II, where positive power corresponds to inverter operation.

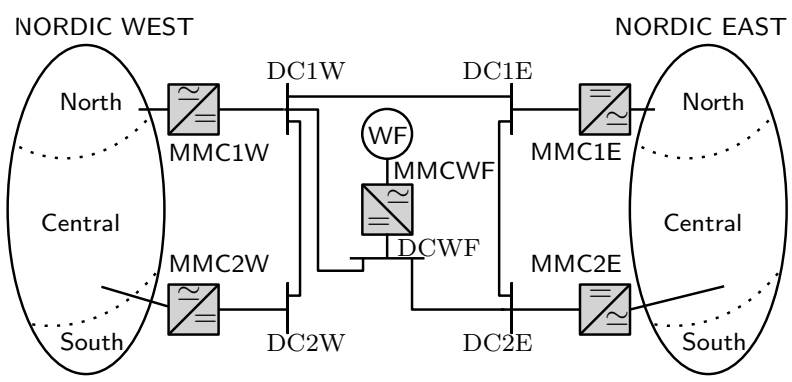

Figure 13: Combined AC and DC system

TABLE I: Cable data

\begin{tabular}{cccc}
\hline From & To & $\mathrm{R}(\Omega)$ & $\mathrm{C}(\mu \mathrm{F})$ \\
\hline DC1W & DC1E & 3,3 & 72 \\
DC1W & DCWF & 3,3 & 72 \\
DC1W & DC2W & 6,6 & 144 \\
DC1E & DC2E & 4,4 & 96 \\
DCWF & DC2E & 6,6 & 144 \\
\hline
\end{tabular}

TABLE II: Operating point

\begin{tabular}{ccc}
\hline MMC & $\mathrm{P}(\mathrm{MW})$ & $V_{d c}(\mathrm{pu})$ \\
\hline MMC1E & -100 & 1,0013 \\
MMC1W & -250 & 1,0003 \\
MMCWF & $-152,7$ & 1,0000 \\
MMC2E & 400 & 0,9949 \\
MMC2W & 100 & 0,9981 \\
\hline
\end{tabular}

\section{B. MMC model and control}

The results presented in this section refer to the following three cases: 


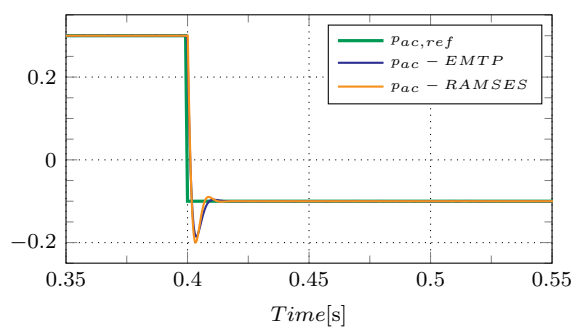

Figure 7: MMC-1 AC power [pu]

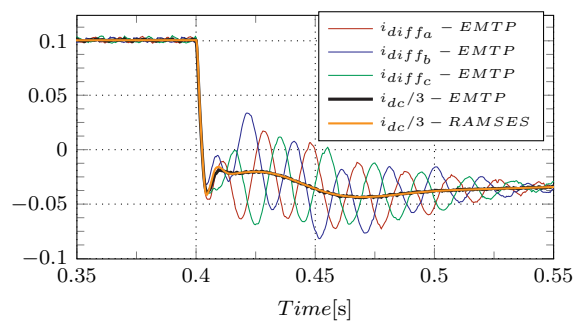

Figure 10: MMC-1 Differential and DC currents $[\mathrm{pu}]$

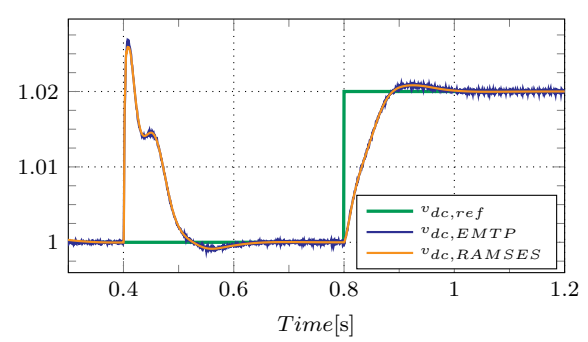

Figure 8: MMC-2 - DC bus voltage [pu]

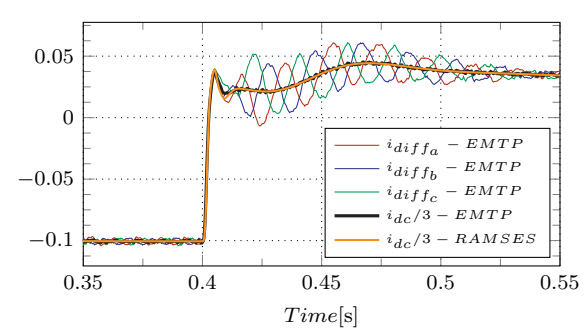

Figure 11: $\mathrm{M}$
currents [pu]

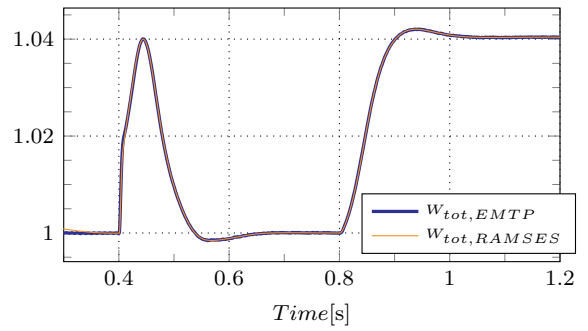

Figure 9: MMC-1 Stored energy [pu]

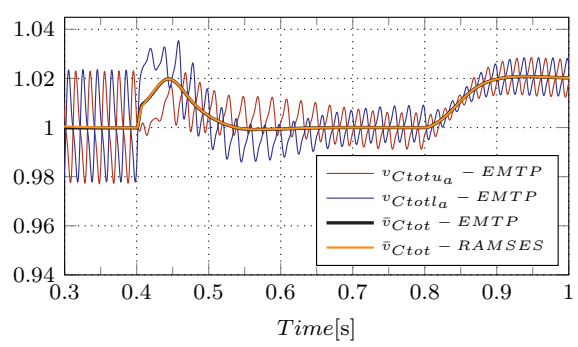

Figure 12: MMC-2 Equivalent arm capacitor voltage $[\mathrm{pu}]$
Case 1: In this case, the proposed MMC model is used (see Fig. 3a) with a constant reference $W_{\text {tot, ref }}$ for the energy controller.

Case 2: The same MMC model as in Case 1, but the square of the DC voltage is used as reference for the energy controller.

Case 3: The MMC is modeled as a classical two-level VSC following the discussion in Section II-D (see also Fig. 3b).

In all cases, each of the MMCs, except MMCWF, is given a power and a DC voltage setpoint (resp. $P_{\text {set }}$ and $v_{d c, s e t}$ ), as well as a droop gain $K_{v}$, and its power reference follows the DC voltage droop characteristic:

$$
P_{a c, r e f}=P_{s e t}+K_{v}\left(v_{d c}-v_{d c, s e t}\right) .
$$

The initial values in Table II are used as the corresponding setpoints. The MMCWF converter imposes constant AC voltage and frequency, thus acting as a slack bus for the wind farm [18].

The parameters of the MMCs are the same as in Section III while $K_{v}$ has been set to $5 \mathrm{pu} / \mathrm{pu}$. A zero reactive power reference is used.

\section{Simulation results}

The scenario considered includes the following events:

- At $t=1 \mathrm{~s}$ the wind farm is suddenly tripped causing a deficit of power in the MTDC grid.

- At $t=20 \mathrm{~s}$ the power references of the MMCs connected to Nordic West are ramped down by $80 \mathrm{MW}$ in a time interval of $5 \mathrm{~s}$.

The system is simulated for $30 \mathrm{~s}$. A time step of $5 \mathrm{~ms}$ (one fourth of a cycle) is used throughout the simulation, except after the loss of the wind farm, when the time step switches briefly to $1 \mathrm{~ms}$ to tackle the discontinuity.

In all cases, the power deficit caused by the loss of the WF power causes the DC voltage to decrease as shown in Fig. 14 for bus DC1W. This causes the DC voltage droop controls of the MMCs to adjust their power to restore the balance in the MTDC grid. This takes some $200 \mathrm{~ms}$ until a new steady state is reached. Since the droop control is a proportional controller, a steady state error remains, which depends on the values of the droop gains. Following the change of power references of MMC1W and MMC2W at $t=20 \mathrm{~s}$, the DC voltages recover near their initial values. The other DC bus voltages follow a similar pattern and are not shown.

A closer look in the first $500 \mathrm{~ms}$ after the first disturbance reveals the differences between the three cases. It can be seen that, although the steady-state is the same, the evolution of Case 1 is faster and much more oscillatory. This is due to the fact that the MMCs do not share their internal energy with the DC grid, whose total capacitance consists only of the cable capacitances. Only small oscillations are present in the response for Case 2, but the average response is the same as for Case 3.

The AC power of MMC1W is shown in Fig. 15, where similar conclusions are drawn. Specifically, the first disturbance causes the rapid change of the MMC AC power. For Cases 2 and 3, the response is similar; some oscillations are present for Case 2. However, the response of Case 1 again exhibits more oscillations.

As expected, the aforementioned events also disturb the power balance of each $\mathrm{AC}$ area, which results in frequency deviations in the East and West systems counteracted by the speed governors. The frequencies of the centers of inertia (COI) of both systems are shown in Fig. 16 only for Case 2 since the difference observed for the other two cases is negligible. Both frequencies start dropping after the loss of the WF power and reach a steady-state after approximately $15 \mathrm{~s}$. The second event causes a further drop of the Nordic West frequency and an increase of the Nordic East frequency 


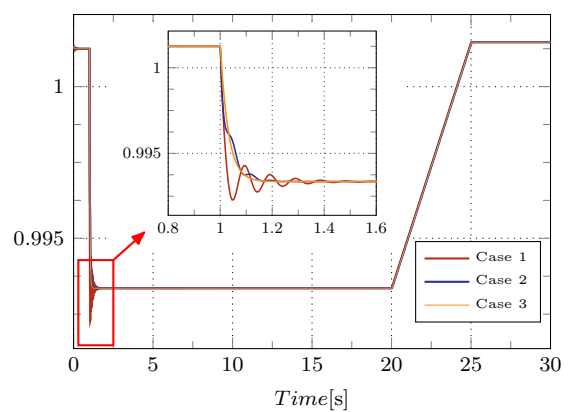

Figure 14: DC voltage at bus DC1W [pu]

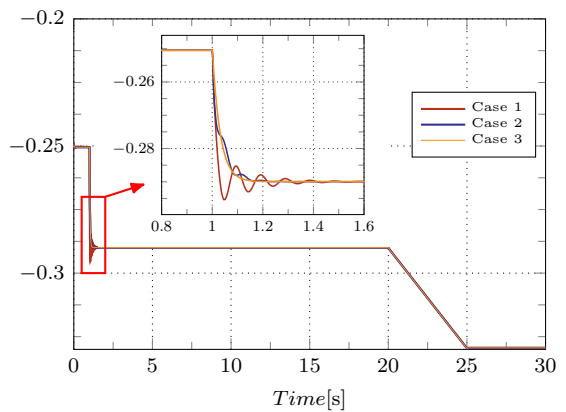

Figure 15: MMC1W Active power [pu]

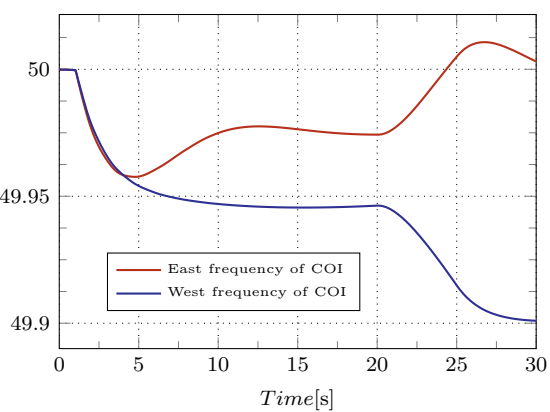

Figure 16: East and West frequency $[\mathrm{Hz}]$ due to the increased power flow from West to East.

\section{CONCLUSiON}

In this paper a simplified model of the MMC has been derived, which can be used for simulation of large systems under the phasor approximation. Reasonable assumptions were made in order to reduce the number of the MMC state variables. An equivalent circuit was derived and a control structure of its state variables was described. The proposed model was validated against an EMT model for a subset of the possible control options and was shown to provide very satisfactory accuracy.

Last but not least, a case study on an MTDC-MMC based grid was presented. A comparison was performed between the proposed MMC simplified model for two energy control options, and the representation of the MMC as a classical twolevel VSC phasor model. The simulation results showed that it cannot be a priori decided whether the classical VSC model is appropriate for the representation of an MMC; this depends on the chosen energy control strategy in the MMC.

The future work envisaged should further investigate and establish the limits of validity of the proposed model, for instance, in terms of control strategies followed, AC system conditions (e.g. low SCR, fault ride through analysis) and simulation time step used.

\section{APPENDIX}

The cable and MMC parameters are listed in Table III.

TABLE III: Parameters

\begin{tabular}{cccc}
\hline \multicolumn{4}{c}{ MMC } \\
\hline$P_{b}$ & $1 \mathrm{GW}$ & $L_{\text {arm }}$ & $48.8924 \mathrm{mH}$ \\
$N$ (SMs) & 400 & $R_{a r m}$ & $1.024 \Omega$ \\
$v_{g}(p h)$ & $320 \mathrm{kV}$ & $C_{\text {tot }}$ & $32.5521 \mu \mathrm{F}$ \\
$v_{d c}$ & $640 \mathrm{kV}$ & $L_{f}$ & $58.6709 \mathrm{mH}$ \\
$R_{f}$ & $0.512 \Omega$ & - & - \\
\hline \hline \multicolumn{4}{c}{ HVDC cable } \\
\hline$R_{d c}$ & $1.54 \Omega$ & $C_{d c}$ & $15 \mu \mathrm{F}$ \\
\hline
\end{tabular}

\section{REFERENCES}

[1] A. Lesnicar and R. Marquardt, "An innovative modular multilevel converter topology suitable for a wide power range," in Proc. IEEE PowerTech Conference, Bologna, June 2003.
[2] S. Teeuwsen, "Simplified dynamic model of a voltage-sourced converter with modular multilevel converter design," in Proc. IEEE PES Power Systems Conference and Exposition, March 2009.

[3] H. Saad, X. Guillaud, J. Mahseredjian, S. Dennetière, and S. Nguefeu, "MMC Capacitor Voltage Decoupling and Balancing Controls," IEEE Transactions on Power Delivery, vol. 30, no. 2, pp. 704-712, Apr. 2015.

[4] S. Liu, Z. Xu, W. Hua, G. Tang, and Y. Xue, "Electromechanical transient modeling of modular multilevel converter based multi-terminal hvdc systems," IEEE Transactions on Power Systems, vol. 29, no. 1, pp. 72-83, Jan 2014.

[5] N.-T. Trinh, M. Zeller, K. Wuerflinger, and I. Erlich, "Generic model of mmc-vsc-hvdc for interaction study with ac power system," Power Systems, IEEE Transactions on, vol. PP, no. 99, pp. 1-8, 2015.

[6] D. Jovcic and A. Jamshidifar, "Phasor Model of Modular Multilevel Converter With Circulating Current Suppression Control," IEEE Transactions on Power Delivery, vol. 30, no. 4, Aug. 2014.

[7] G. Bergna Diaz, J. A. Suul, and S. D'Arco, "Small-signal state-space modeling of modular multilevel converters for system stability analysis," in Energy Conversion Congress and Exposition (ECCE), 2015 IEEE, Sept 2015, pp. 5822-5829.

[8] H. Saad, S. Dennetière, J. Mahseredjian, P. Delarue, X. Guillaud, J. Peralta, and S. Nguefeu, "Modular multilevel converter models for electromagnetic transients," IEEE Transactions on Power Delivery, vol. 29, no. 3, pp. 1481-1489, June 2014.

[9] H. Saad, "Modélisation et simulation d'un liason hvdc de type vscmmc," Ph.D. dissertation, École Polytechnique de Montréal, March 2015.

[10] P. Rault, F. Colas, X. Guillaud, and S. Nguefeu, "Method for small signal stability analysis of VSC-MTDC grids," IEEE PES General Meeting, vol. 3, Jul. 2012.

[11] S. Samimi, F. Gruson, X. Guillaud, and P. Delarue, "Control of dc bus voltage with a modular multilevel converter," in Proc. IEEE PowerTech, Eindhoven, June 2015.

[12] P. Delarue, F. Gruson, and X. Guillaud, "Energetic macroscopic representation and inversion based control of a modular multilevel converter," in 15th European Conference on Power Electronics and Applications (EPE), Sept 2013.

[13] P. Aristidou, D. Fabozzi, and T. Van Cutsem, "Dynamic simulation of large-scale power systems using a parallel Schur-complement-based decomposition method," IEEE Transactions on Parallel and Distributed Systems, vol. 25, no. 10, pp. 2561-2570, 2014.

[14] P. Aristidou, L. Papangelis, X. Guillaud, and T. Van Cutsem, "Modular modelling of combined AC and DC systems in dynamic simulations," in Proc. IEEE PowerTech, Eindhoven, Jun. 2015.

[15] CIGRE brochure 604, "Guide for the development of models for hvdc converters in a hvdc grid," WG B4-57, 2014.

[16] J. Mahseredjian, S. Dennetière, L. Dubé, B. Khodabakhchian, and L. Gérin-Lajoie, "On a new approach for the simulation of transients in power systems," Electric Power Systems Research, vol. 77, no. 11, pp. 1514-1520, Sep. 2007.

[17] IEEE PES Task Force, "Test Systems for Voltage Stability Analysis and Security Assessment," Tech. Rep., 2015. [Online]. Available: http://resourcecenter.ieee-pes.org/pes/product/technical-reports/PESTR19

[18] L. Xu, L. Yao, and C. Sasse, "Grid Integration of Large DFIG-Based Wind Farms Using VSC Transmission," IEEE Transactions on Power Systems, vol. 22, no. 3, pp. 976-984, Aug. 2007. 